on the experience of children, teachers, and emergency services regarding management of prolonged convulsive seizures occurring at school. (Cross JH, Wait S, Arzimanoglou A, et al. Are we failing to provide adequate rescue medication to children at risk of prolonged convulsive seizures in schools? Arch Dis Child 2013 Oct;98(10):777-80). (Response: Professor J Helen Cross. E-mail: h.cross@ucl.ac.uk).

COMMENT. In the past, a seizure lasting 30 min or longer was considered status epilepticus. Currently, experts classify any episode of seizure activity lasting $5 \mathrm{~min}$ or longer as status epilepticus. Once seizures persist for 5 to $10 \mathrm{~min}$, they are unlikely to stop without treatment. Pre-hospital treatment with benzodiazepines will reduce seizure activity whereas delayed treatment is less successful, with risk of subsequent prolonged seizure activity, memory deficit, and learning difficulties. (Pellock JM. J Child Neurol 2007 May;22(5 Suppl):9S-13S).

A $>15$ min duration of a febrile seizure is one criterion for the definition of a complex seizure, but a recent FEBSTAT study supports a redefining of simple vs complex seizure, limiting the duration of a simple febrile seizure to no longer than $10 \mathrm{~min}$ (Hesdorffer DC, et al. Ann Neurol 2011 Jul;70(1):93-100).

Spontaneous seizures and febrile seizure duration. Data in support of a shorter 5-10 min cut off for a simple FS were obtained in a comparative study of 86 consecutive patients with FSs of short and long duration (Millichap JG, et al. Neurology 1960 Jul;10:643-53; Millichap JG. Febrile Convulsions. New York: Macmillan, 1968, pp 1013). In 38 patients with FS $<5$ min duration, 7.9\% developed spontaneous seizures; in 21 with FS 5-10 min duration, 9.5\%; in 14 with FS 10-20 min duration, 14\%; and in 13 with FS $>20$ min duration, $38 \%$ had spontaneous seizures. The difference in spontaneous seizure incidence in patients with FS of 5 and 10 min duration was not significant whereas that between the 10 and $>20$ min FS duration was very significant. The prompt treatment within 5-10 min of onset of a convulsive seizure (febrile or non-febrile) is recommended, using an age-appropriate benzodiazepine preparation (rectal diazepine. intranasal lorazepam, or buccal midazolam). (Sofou K, et al. J Child Neurol 2009 Aug;24(8):918-26). For the optimal outcome of children at risk of prolonged convulsive seizures, rescue treatment for administration at home or in the school should be available.

\title{
PROGNOSIS OF EARLY ONSET ABSENCE EPILEPSY
}

Investigators from University of Chieti and several other centers in Italy conducted a multicenter retrospective 36-month follow-up study of the electroclinical course of epilepsy in all children with typical absence seizures (TAS) starting in the first 3 years of life. Two groups of patients were compared: 1) 111 who fulfilled Panayiotopoulos's criteria for childhood absence epilepsy (CAE) classified as having pure early onset absence epilepsy (P-EOAE), and 2) 77 who did not satisfy the criteria and were classified as nonpure EOAE (NP-EOAE). The 2 groups were also stratified according to the number of antiepileptic drugs used to obtain initial seizure control.

Patients with pure EOAE showed earlier initial seizure control $(p=0.030)$ and better seizure-freedom $(\mathrm{p}=0.004)$ than those with NP-EOAE. P-EOAE patients had no mutation in SLC2A1 gene and no abnormal neuroimaging. Among the NP-EOAE 
patients, those receiving tritherapies showed increased risk of structural brain abnormalities $(\mathrm{p}=0.001)$ or SLC2A1 mutations $(\mathrm{p}=0.001)$ but fewer myoclonic features $(\mathrm{p}=0.031)$ and worse seizure-free survival $(\mathrm{p}=0.047)$ than those treated with mono-and biotherapy. Children with NP-EOAE had an increased risk of relapse during follow-up compared to P-EOAE patients. (Agostinelli S, Accorsi P, Beccaria F, et al. and on behalf of the Societa Italiana Neurologia Pediatrica Collaborative Working Group. Epilepsia 2013 Oct;54(10):1761-70). (Response: Dr Alberto Verrotti, Department of Pediatrics, University of Chieti, Italy. E-mail: averrott@unich.it).

COMMENT. Children $<3$ years old with early onset TAS who meet the modified Panayiotopoulos's criteria for childhood absence epilepsy (CAE) have a good prognosis, whereas those not meeting the criteria have an increased risk of relapse at long-term follow-up.

Panayiotopoulos's definition of CAE (Panayiotopoulos CP. Epilepsia 2008 Dec;49(12):2131-9) is abridged as follows: 1) age at onset between 4 and 10 years (modified to within the first 3 years for this study); 2) normal neurologic exam; 3) brief (4-20 sec) and frequent (many per day); 4) EEG 3-4 Hz spike and slow-wave complexes. Exclusion criteria include 1) other types of seizure; 2) eyelid myoclonia, perioral myoclonia, head and limb myoclonic jerks; 3) EEG polyspikes; 4) photic precipitation of clinical seizures.

\section{TRANSITION FROM PEDIATRIC TO ADULT HEALTH CARE FOR DRAVET SYNDROME PATIENTS}

Investigators from University Hospital of Rennes; Necker Hospital, Paris; and University Paris Descartes, France used a questionnaire to study the transition and transfer from pediatric to adult health-care system in patients with Dravet syndrome and their families. The diagnosis of Dravet syndrome was made during the first 2 years and was followed by a long follow-up in the pediatric health-care. A response rate of $85 \%$ was obtained from 60 families, and $61 \%$ experienced a transfer. Positive factors for a smooth transition included 1) the quality of transition preparation $(p<.000001), 2)$ a longer duration of follow-up by the same child neurologist $(p<.001), 3)$ the availability of the child neurology staff $(\mathrm{p}<.01), 4)$ transfer into adult health-care after the age of 18 $(\mathrm{p}<.01)$, and 5) a stable medical condition before transfer $(\mathrm{p}<.05)$. The age of transfer (18.7 +/ 4 years) was close to the legal age of adulthood, and the association of mental retardation with severe epilepsy had little impact on transfer age. All families reported a positive experience in the pediatric health care system. Child neurologists were considered as welcoming, available, and helpful. Almost all patients transferred reported no gap in the process. Their experience in the adult health-care system was similar to pediatric care. Only $9 \%$ patients contacted their child neurologist after the transfer, and $79 \%$ continued follow-up with the same neurologist. Preparation for transfer began an average of 1 year before transition, which is shorter than that generally recommended for chronic illnesses. (Kuchenbuch M, Chemaly N, Chiron C, Dulac O, Nabbout R. Transition and transfer from pediatric to adult health care in epilepsy: A families' survey on Dravet syndrome. Epilepsy Behav 2013 Oct;29(1):161-5). (Response: Dr Rima Nabbout, Hospital Necker Enfants Malades, Paris. E-mail: rimanabbout@yahoo.com). 